\title{
Análisis Bifactorial de la Escala de Participación Escolar en una Muestra de Estudiantes Chilenos
}

\author{
Bifactor Analysis of the School Participation Scale in a Chilean Student Sample
}

\author{
Claudia P. Pérez-Salas ${ }^{1}$, David Sirlopú ${ }^{2}$, Rubia Cobo ${ }^{3}$ y Alia Awad ${ }^{4}$
}

\begin{abstract}
Resumen
La escala de participación escolar fue diseñada por John-Akinola y Nic-Gabhainn (2014) para evaluar la participación escolar desde la percepción de los propios estudiantes. La presente investigación tuvo como objetivo principal conocer las propiedades psicométricas de este cuestionario, a través de una aplicación colectiva a 1428 estudiantes de escuelas secundarias del centro-sur de Chile (53\% mujeres), con edades entre 12 y 21 años $(\mathrm{M}=15.59 ; \mathrm{DE}=1.52)$. El análisis factorial confirmatorio mostró un buen ajuste a los datos para el modelo bifactorial probado y los coeficientes de consistencia interna de las escalas fueron adecuados. Los resultados sugieren que la versión en español del cuestionario de participación escolar es un instrumento válido y confiable para evaluar este constructo en estudiantes chilenos.
\end{abstract}

Palabras clave: modelado bifactorial, participación escolar, confiabilidad, validez

\begin{abstract}
The school participation scale was developed by John-Akinola and Nic-Gabhainn (2014) to assess student's participation at school from his/her perspective. This investigation aimed to estimate the psychometrics properties of this questionnaire in a sample of 1428 secondary students from Chile (53\% females), with ages from 12 to 21 years old $(M=15.59 ; S D=1.52)$. Bifactor analyses showed best fit for the proposed hierarchical structure, and the internal consistency coefficients were adequate. Results suggest that the Chilean version of the school participation scale is a valid and reliable instrument for assessing this construct in school aged children in Chile.
\end{abstract}

Keywords: bifactor modeling, school participation, reliability, validity

\footnotetext{
${ }^{1}$ Doctora en Psicología, Departamento de Psicología, Universidad de Concepción, Barrio Universitario s/n, Departamento de Psicología, $2^{\circ}$ piso, Facultad de Ciencias Sociales, Concepción Chile. Tel.: +56-41-2204301. Correo: cperezs@udec.cl

${ }^{2}$ Doctor en Psicología, Facultad de Psicología, Universidad del Desarrollo, Concepción, Chile.

${ }^{3} \operatorname{Doctora}(c)$ en Psicología, Departamento de Psicología, Universidad de Concepción, Chile.

${ }^{4}$ Magíster en Psicología, Departamento de Psicología, Universidad de Concepción, Chile.
} 


\section{Introducción}

La participación, en términos generales, se puede definir como la posibilidad y la acción de ser tomado en cuenta e incidir en el espacio social en el que se convive (Cornejo \& Redondo, 2001). Cuando se habla de población infantil y juvenil, la participación es descrita como un proceso mediante el cual los miembros de estos grupos etarios colaboran entre sí en asuntos relacionados con su bienestar colectivo (Chawla, 2001, citado en John-Akinola, Gavin, O'Higgins, Nic Gabhainn, 2014). Por su parte, la participación escolar cuenta con un enfoque más acotado. Esta se refiere al conjunto de prácticas cuyo objetivo es alentar la expresión de opiniones, la toma de decisiones democráticas o conjuntas y el involucramiento en los procesos de decisión de los alumnos dentro de los colegios (Griebler, Rojatz, Simovska, \& Forster, 2012; Simovska, 2017).

La escuela es un espacio privilegiado de participación, tanto por las interacciones cotidianas que allí se dan, como por la oportunidad de transmitir aprendizajes y desarrollar actitudes y habilidades que favorecen la convivencia democrática y la participación social activa (Cornejo \& Redondo, 2001). Por tanto, la promoción de la participación escolar es importante para el desarrollo integral de ciudadanas y ciudadanos con sentido de responsabilidad cívica y social. La participación también contribuye al desarrollo personal, bienestar y salud mental de los estudiantes, ya que un mayor grado de participación e identificación con el establecimiento educacional, se correlaciona positivamente con una mejor autoestima, mayor sentimiento de autonomía y autocontrol, mayor capacidad de establecer relaciones de apoyo y colaboración con otras personas, desarrollo de interacciones sociales positivas, mayor grado de satisfacción con la vida y menor probabilidad de incurrir en conductas antisociales o de riesgo (de Róiste, Kelly, Molcho, Gavin, \& Gabhainn, 2012; Finn \& Rock, 1997; John-Akinola \& Nic-Gabhainn, 2014)

Los centros escolares que facilitan las instancias de participación favorecen la interacción, los vínculos de confianza y la comunicación entre los distintos estamentos de la comunidad educativa, mejorando su desempeño como organización y a la vez, contribuyendo al desarrollo de los procesos educativos y al rendimiento académico. Esto se logra, de varias maneras: un clima escolar participativo se relaciona con mayor sentido de pertenencia, con mayor nivel de compromiso y disposición de los estudiantes, docentes y apoderados hacia los procesos de enseñanza-aprendizaje y hacia los logros académicos, junto con mayor asistencia escolar, menores tasas de deserción y mayor perseverancia por parte de los estudiantes (Fullarton, 2002). El desarrollo de una cultura escolar más justa y participativa requiere de algunos factores como la existencia de actividades de encuentro, espacios disponibles para organizar actividades, disposición del establecimiento educacional para acoger inquietudes $\mathrm{y}$ sugerencias, y la valoración de los docentes y directivos de las iniciativas y contribuciones que realizan todos los integrantes de la comunidad educativa (Libbey, 2004).

\section{Participación escolar en Chile}

El Ministerio de Educación de Chile considera la participación escolar como un eje fundamental para el desarrollo integral de los estudiantes. Desde el 2013 se incorporó junto con las mediciones del Sistema de Medición de la Calidad de la Educación (SIMCE), mediciones de "Indicadores de Desarrollo Personal y Social". Estos corresponden a aspectos no académicos que favorecen una educación más integral en los estudiantes y que deben ser fomentados por cada centro educativo.

Uno de los indicadores es la "Participación Escolar y Formación ciudadana". Este se relaciona con el sentimiento de identificación, pertenencia y vinculación del estudiante con la institución escolar y la sociedad en general. La medición de este indicador considera la dimensión de las actitudes y percepciones de los estudiantes y apoderados sobre el grado en que el colegio promueve la participación, la vida democrática y el compromiso de los miembros de la comunidad educativa y clasifica las respuestas en tres niveles: alto, medio y bajo (Ministerio de Educación, 2014). Los resultados de este indicador evidencian una brecha de género, pues las mujeres disminuyeron progresivamente el nivel de 
participación a medida que aumentaba el grado escolar. Según la distribución socioeconómica, se observa que en segundo medio, el $62 \%$ de los escolares pertenecientes al nivel socioeconómico más alto reportó un nivel alto de participación, mientras que entre los escolares de nivel socioeconómico bajo, dicho nivel alto de participación fue 48\% (Ministerio de Educación de Chile, 2017).

Resultados similares fueron encontrados el 2013 respecto a las diferencias en los niveles de "Vida democrática" y en la "Participación e Identificación de los estudiantes con su establecimiento" según el grupo socioeconómico. Específicamente se reportó mayor participación escolar en los niveles socioeconómicos altos y establecimientos más pequeños (con uno o dos cursos por grado). En este estudio, además, se encontró una relación positiva de la participación con el rendimiento académico. Así, los puntajes en la prueba SIMCE de lenguaje y de matemática fueron más altos en los establecimientos con una mayor participación escolar que en el resto de los establecimientos, independiente del grupo socioeconómico (Ministerio de Educación de Chile, 2014).

La implicancia futura de la participación escolar en la participación política ha sido estudiada por Rodríguez, Kohen y Messina (2016). Estos autores evaluaron la influencia de la participación escolar en la intención de participación política en el futuro en acciones convencionales (v.g., votar o afiliarse a un partido político) y no convencionales (v.g., asistir a manifestaciones, adherir a boicots o realizar huelgas) de 302 estudiantes entre 13 y 18 años. Los resultados mostraron que ejercer un cargo escolar influye significativamente en la formación democrática y la intención de participar políticamente en la sociedad a través de acciones no convencionales, tales como participar en huelgas. Si bien, la participación escolar predijo mejor este tipo de acciones, no influyó significativamente en acciones políticas convencionales como la intención de votar, pero sí en la intención de afiliarse a un partido político.

\section{La Escala de Participación Escolar}

Los antecedentes antes expuestos ponen de relieve la importancia de estudiar y promover la participación en la escuela. A pesar de esto, existen escasos instrumentos para medir este constructo. Una excepción lo constituye la escala de participación escolar de John-Akinola y NicGabhainn (2014). John-Akinola y sus colaboradores (2014) estudiaron la relación entre la participación de escolares y sus apoderados en la salud de la comunidad escolar. Para lograr dicho fin, estos autores emplearon un diseño de métodos mixtos. En la fase cualitativa entrevistaron a escolares y sus padres con el fin de conocer sus nociones acerca de la participación escolar. Así emergieron cuatro dimensiones de la: participación en la toma de decisiones y normas escolares; participación en eventos especiales dentro de la escuela; participación en actividades regulares extra-académicas organizadas por el colegio; y percepción positiva de la participación escolar. En la fase cuantitativa, se elaboraron distintos ítems asociados a cada una de estas cuatro dimensiones (John-Akinola et al., 2014).

La dimensión que alude a la toma de decisiones y normas escolares se vincula con una forma preparatoria de involucrar a los alumnos en la toma de decisiones conjuntas y democráticas dentro de la sala de clases o en diversas actividades escolares (Griebler et al., 2017; Simovska, 2004). La participación en eventos especiales de la escuela y en actividades regulares extra-académicas son concebidas como aquellas acciones en las que los escolares participan día a día en sus establecimientos. Estas actividades debieran ser estimulantes en términos de su atractivo, pero también de formación, puesto que pueden contribuir en el desarrollo cognitivo e incremento de las capacidades para el aprendizaje de los alumnos (Simovska, 2012). Finalmente, la percepción positiva de la participación escolar identifica si esta es genuina y real o más bien simbólica. Para John-Akinola y Nic-Gabhainn (2014) estas cuatro dimensiones se constituyen en pilares fundamentales de la participación escolar.

La investigación de estos autores presenta una definición amplia sobre la participación escolar enfocada en la perspectiva de los propios niños y niñas. Comprender cómo se presenta este fenómeno desde la mirada de los estudiantes es un aporte valioso para la investigación en este contexto. Sin embargo, en su estudio de validación no se presentaron análisis 
psicométricos de la estructura factorial de la Escala de Participación Escolar (en adelante EPE), ya que solo se reportaron índices de consistencia interna y una pequeña revisión por expertos para el análisis de la validez de contenido. En tal sentido y considerando la relevancia del constructo, el presente estudio tiene como objetivo evaluar la estructura factorial de la EPE en una muestra de estudiantes chilenos. Se hipotetiza una estructura bifactorial en este cuestionario, puesto que la escala considera cuatro dimensiones, además de la posibilidad de obtener un puntaje total que refleja un factor general de participación. Según Reise, Moore y Haviland (2010) los análisis factoriales de segundo orden y bifactoriales son los únicos modelos que reconocen la multidimensionalidad y simultáneamente retienen la existencia de un modelo general, pero los bifactoriales serían más apropiados cuando las escalas entregan un puntaje total interpretable y subdominios importantes para la comprensión del fenómeno evaluado (Brown, 2014)

\section{Análisis Bifactorial: Aspectos Generales}

En psicometría se proponen varios enfoques para el análisis de dimensiones subyacentes o factores de una escala de medición. Entre ellos se encuentran el análisis factorial exploratorio (AFE), el análisis factorial confirmatorio (AFC), el análisis factorial confirmatorio de segundo orden (AFCSO) y el análisis bifactorial (Brown, 2014). Este último, ha sido uno de los modelos estadísticos menos empleados en las ciencias sociales, pese a que fue propuesto hace más de 70 años por Holzinger y Swineford (1937).

El modelo bifactorial es un tipo de análisis factorial confirmatorio de segundo orden que asume la existencia de un factor general que explica la covarianza de todas las medidas observadas (Brown, 2014) y de forma simultánea presenta varios factores de primer orden que median y explican una parte de los mismos ítems (Chen, West, \& Sousa, 2006). Así, es posible especificar los efectos directos de los factores de primer orden y del factor general (de segundo orden), sin estar necesariamente correlacionados. Es apropiado para las escalas de medida que entregan un puntaje total interpretable, pero que poseen subdominios que poseen un valor sustantivo para la investigación (Brown, 2014).

Distintos autores (Chen et al., 2006; Reise et al., 2010) han resumido las ventajas del modelo bifactorial sobre el AFCSO. La principal ventaja es que el análisis bifactorial permite observar directamente hasta qué punto un ítem o escala (la variable observada) refleja un rasgo objetivo común (es decir, un factor general) y, simultáneamente, hasta qué punto puede reflejar un subrasgo (específico del dominio). En consecuencia, el modelo bifactorial permite retener un único factor latente común, pero también controla la varianza que surge debido a factores comunes adicionales.

Una segunda ventaja del modelo bifactorial es que en el modelo de AFCSO no es posible observar las relaciones directas entre las variables observadas y el factor general, sino más bien un "efecto indirecto" o una "relación mediada" a través de los factores de primer orden. Por lo tanto, para estimar la varianza atribuible al factor general, la carga de la variable observada en el factor específico del dominio debe ser multiplicada por la carga del factor específico del dominio sobre el factor general (Chen et al., 2006). Por el contrario, el modelo bifactorial proporciona información sobre todas las cargas de factores $\mathrm{y}$ permite identificar si un factor específico del dominio contribuye de manera única a la predicción de criterios externos (Chen et al., 2006). Debido a que en un modelo bifactorial, los factores generales y específicos del dominio son ortogonales, incluso una simple inspección de las cargas de los ítems en los factores de segundo y primer orden resulta informativa.

Dado que el modelo de AFCSO está anidado en el modelo bifactorial no se presentan tales restricciones. Este último puede ser usado como un modelo base para comparar el ajuste del modelo mientras el modelo se vuelva más restringido, lo que constituye una tercera ventaja de lo modelo bifactorial sobre el AFCSO (Brown, 2014). Por ejemplo, en un modelo de AFCSO se asume que las correlaciones entre los factores de primer orden ocurren porque tienen una causa común (es decir, el factor general). Por lo tanto, la observación de bajas cargas en un factor específico del dominio y altas cargas de los 
factores específicos en un factor general pueden sugerir que estas variables se explican mejor por un factor general y no constituyen un factor de dominio específico. Así, si los ítems reflejan principalmente el factor general y tienen cargas bajas en los factores de primer orden, las subescalas tienen poco sentido.

Los aportes del modelo bifactorial en la comprensión y medición de distintos constructos de la psicología ha comenzado a difundirse. Así, el modelo bifactorial ha sido empleado para evaluar medidas de inteligencia (Cucina \& Byle, 2017; Gignac, 2016; Schult \& Sparfeldt, 2016), personalidad (Gade, Schermelleh-Engel, \& Klein, 2017), autoestima (Michaelides et al., 2016), esperanza (Brouwer, Meijer, Weekers, \& Baneke, 2008), calidad de vida (Chen et al., 2006) y bienestar (Chen, Jing, Hayes, \& Lee, 2013).

En el contexto educativo es importante la creación e implementación de escalas que evalúen los fenómenos propios de la vida de los estudiantes, los docentes y del entorno en donde estos se desenvuelven. En este caso, el uso del modelo bifactorial ha sido implementado para la comprensión de varios constructos tales como el compromiso escolar (Steffansson, Gestsdottir, Geldhof, Skulason, \& Lerner, 2016), la percepción de la calidad de la instrucción (Scherer, Nilsen, \& Jansen, 2016) o la interacción docente-estudiante (Hindman, Pendergast, \& Gooze, 2016).

Considerando los aportes metodológicos y prácticos del modelo bifactorial en el análisis de escalas de medición de variables multidimensionales en el entorno educativo, el presente estudio pretende evaluar la estructura bifactorial de la EPE (John-Akinola \& NicGabhainn, 2014) en una muestra de estudiantes chilenos.

\section{Método}

\section{Participantes}

Se realizó un muestreo no aleatorio intencional con el cual se encuestaron a 1428 estudiantes de escuelas secundarias del centro-sur de Chile (754 mujeres y 671 hombres); tres participantes no clasificaron su sexo con ninguna de las alternativas anteriores. Sus edades estuvieron comprendidas entre los 12 y 21 años $(M=15.59 ; D E=1.52)$ y cursaban octavo básico (el último año de educación básica en Chile) (15.8\%), primero medio (22.5\%), segundo medio $(22.7 \%)$, tercero medio $(21.4 \%)$ y cuarto medio $(17.6 \%)$,

\section{Medidas}

La escala de participación escolar (JohnAkinola \& Nic-Gabhainn, 2014) se compone de 25 ítems organizados en cuatro dimensiones: (1) participación de las decisiones y las normas escolares (6 ítems: "Los alumnos participan en la elaboración del reglamento del colegio), (2) participación en eventos escolares tales como "Día del deporte" (6 ítems: "Los alumnos participan de la planificación de eventos escolares), (3) participación en actividades escolares extra-académicas tales como educación física, artes, música, deportes, teatro, viajes escolares (7 ítems: "Me entretengo realizando estas actividades extraprogramáticas") y (4) percepción positiva de la participación escolar (6 ítems: "En mi colegio, todos los alumnos tienen la posibilidad de participar"). Se utilizó una escala de respuesta de tipo Likert de cinco puntos (1= nunca; $5=$ siempre). En cuanto, a la fiabilidad de las dimensiones reportada por sus autores esta fue heterogénea ( $\alpha$ entre .60 y .70).

\section{Procedimiento}

Para la adaptación y validación al español de la EPE se siguió las recomendaciones de Muñiz, Elosua y Hambleton (2013). Primero se intentó conseguir el consentimiento de los autores para el uso del instrumento. Sin embargo, no fue posible obtener respuesta de ninguno de ellos. Por tanto, ya que es un instrumento completamente publicado en su artículo original, se procedió con las siguientes etapas. Segundo, se realizaron las adecuaciones idiomáticas del inglés al español con dos traductores independientes. Tercero, las traducciones fueron revisadas por dos psicólogos quienes hicieron algunas observaciones para adaptar palabras o frases bien traducidas, pero que podrían tener dificultades de comprensión en la cultura de habla española y en el grupo etario elegido. La versión definitiva en español fue enviada a otro traductor para que la transcribiera literalmente al inglés. Al comparar la versión 
original en inglés con la retro-traducida, no se observaron mayores diferencias.

Con respecto al trabajo de campo, los coordinadores del proyecto se contactaron telefónicamente con los directivos de los colegios que habían sido previamente seleccionados, para informarles de las características del estudio. Aquellos directores que aceptaron participar, debieron completar una autorización institucional. Los encuestadores se reunieron con los profesores de cada curso y se les contó a los alumnos de qué trataba el estudio. Los escolares que estuvieron dispuestos a responder el cuestionario, debían traer un consentimiento informado firmado por alguno de sus padres o apoderado. Solo los escolares que trajeron al colegio los consentimientos firmados pudieron participar del estudio. Además, los escolares debieron firmar un asentimiento informado. En retribución a su tiempo, se les entregó una entrada al cine (aproximadamente USD 4).

\section{Análisis de Datos}

Se utilizó el programa estadístico JASP versión 0.8.4 (JASP Team, 2018) para el análisis de las estadísticas descriptivas de la muestra normativa chilena y para la estimación de la consistencia interna de la prueba con el coeficiente omega de Mc Donald. Se utilizó este coeficiente debido a la mayor estabilidad de su cálculo con datos que no son continuos (VenturaLeón \& Caycho-Rodríguez, 2017). Por otra parte, los análisis factoriales confirmatorios de segundo orden y bifactoriales se realizaron con la finalidad de evaluar la estructura subyacente de la EPE y su ajuste comparativo. Para ambos casos se empleó el método WLSMV (weighted least squared means and variance adjusted) utilizando el software MPlus 7.11 (Muthén \& Muthén, 2013).

\section{Resultados}

En la Tabla 1 se presentan las medidas de tendencia central y de dispersión obtenidas de la muestra de participantes chilenos. En general, los puntajes reportan niveles altos de participación para las cuatro dimensiones (entre 20 y 22 puntos). Para la confiabilidad se realizó el análisis de confiabilidad omega de McDonald, los resultados muestran que tanto las dimensiones de la EPE como su puntuación global presentan altos niveles de consistencia interna. La literatura establece que valores adecuados del omega deben ser superiores a .70 y la correlación corregida ítem-total sea superior a .30 (Luján-Tangarife \& Cardona-Arias, 2015).

La Tabla 2 presenta las correlaciones corregidas ítem-total, donde se reflejan niveles de asociación adecuados en todos los ítems del cuestionario con respecto a sus dimensiones.

\section{Análisis Factorial Confirmatorio Modelo con Factores de Primer y Segundo Orden}

Con el objetivo de evaluar la estructura factorial de la EPE se realizó un análisis factorial confirmatorio considerando cuatro dimensiones de primer orden y un factor general de segundo orden. En la Tabla 3 se presentan los resultados del ajuste obtenido para este modelo. Se puede observar que los valores de los índices de ajuste no se encuentran dentro de lo esperado teóricamente. Se presenta un valor significativo del test Chi-Cuadrado, el error medio cuadrático RMSEA es superior al rango recomendado .05.08 , esperándose valores inferiores a este criterio y el índice de ajuste comparativo no se encuentra dentro del rango >.95 (Geiser, 2012).

Tabla 1. Estadísticas descriptivas de las dimensiones de la escala de participación escolar para la muestra normativa chilena

\begin{tabular}{|c|c|c|c|c|c|c|}
\hline Dimensiones & $\begin{array}{l}\text { Puntaje } \\
\text { mínimo }\end{array}$ & $\begin{array}{l}\text { Puntaje } \\
\text { Máximo }\end{array}$ & $\begin{array}{c}\text { Media } \\
\text { (Puntaje } \\
\text { total) }\end{array}$ & $\begin{array}{c}\text { Desviación } \\
\text { Estandar }\end{array}$ & Omega & $\begin{array}{l}\text { Cantidad } \\
\text { de ítems }\end{array}$ \\
\hline $\begin{array}{l}\text { Participación de las decisiones y } \\
\text { normas }\end{array}$ & 6.00 & 30.00 & 19.94 & 5.04 & .853 & 6 \\
\hline $\begin{array}{l}\text { Participación en los eventos } \\
\text { escolares }\end{array}$ & 6.00 & 30.00 & 22.01 & 4.89 & .835 & 6 \\
\hline $\begin{array}{l}\text { Participación en las actividades } \\
\text { escolares (extra-académicas) }\end{array}$ & 7.00 & 35.00 & 23.70 & 6.73 & .866 & 7 \\
\hline Percepción positiva de participación & 6.00 & 30.00 & 22.36 & 5.06 & .877 & 6 \\
\hline
\end{tabular}


Tabla 2. Correlación ítem-test corregida de la escala de participación escolar

\begin{tabular}{|c|c|c|c|c|}
\hline Ítems & DN & $\mathrm{EE}$ & $\mathrm{AE}$ & PP \\
\hline 1 & $.468^{* * *}$ & & & \\
\hline 2 & $.735^{* *}$ & & & \\
\hline 3 & $.749^{* *}$ & & & \\
\hline 4 & $.734^{* *}$ & & & \\
\hline 5 & $.699^{* *}$ & & & \\
\hline 6 & $.374^{* *}$ & & & \\
\hline 7 & & $.593^{* *}$ & & \\
\hline 8 & & $.676^{* *}$ & & \\
\hline 9 & & $.620^{* * *}$ & & \\
\hline 10 & & $.482^{* * *}$ & & \\
\hline 11 & & $.673^{* *}$ & & \\
\hline 12 & & $.573^{* *}$ & & \\
\hline 13 & & & $.697^{* *}$ & \\
\hline 14 & & & $.766^{* *}$ & \\
\hline 15 & & & $.726^{* *}$ & \\
\hline 16 & & & $.585^{* *}$ & \\
\hline 17 & & & $.465^{* *}$ & \\
\hline 18 & & & $.468^{* *}$ & \\
\hline 19 & & & $.721^{* *}$ & \\
\hline 20 & & & & $.571^{* *}$ \\
\hline 21 & & & & $.738^{* *}$ \\
\hline 22 & & & & $.692^{* *}$ \\
\hline 23 & & & & $.737^{* *}$ \\
\hline 24 & & & & $.722^{* *}$ \\
\hline 25 & & & & $.622^{* *}$ \\
\hline
\end{tabular}

Nota. N=1428; DN: Participación de las Decisiones y Normas escolares; EE: participación en los Eventos Escolares; AE: Participación en las Actividades Escolares (extra-académicas); PP: Percepción Positiva de la participación;** $p<.01$.

Tabla 3. Estimaciones del modelo con cuatro factores de primer orden y uno de segundo orden para la escala de participación escolar

\begin{tabular}{lcccccc}
\hline $\mathrm{X}^{2}$ & $\mathrm{~d} f$ & RMSEA & $(90 \% \mathrm{CI})$ & WRMR & CFI & TLI \\
\hline $4422.529^{*}$ & 271 & .102 & $.099-.105$ & 3.193 & .894 & .883
\end{tabular}

Nota. $\mathrm{N}=1428 ; * p<.01$

En la Tabla 4 se reportan las cargas factoriales de los cuatro factores propuestos para el análisis presentaron cargas significativas en el factor de segundo orden. En general, los ítems presentaron cargas adecuadas en cada factor, confirmándose las dimensiones de (a) participación de las decisiones y normas escolares, (b) participación en las actividades escolares (extra-académicas), (c) participación en los eventos escolares, y (e) percepción positiva de la participación (Ver Tabla 4).

\section{Modelo Bifactorial}

Se analizó un modelo bifactorial, considerando los mismos factores de primer orden que en el AFCSO recién realizado y además un factor de segundo orden (bifactor) donde cada ítem también se encontraba subsumido (Factor G). Como se observa en la Tabla 5, los resultados del modelo bifactorial mostraron indicadores de ajuste adecuados con valores RMSEA y CFI dentro de lo esperado teóricamente (Geiser, 2012). Si bien el valor del chi cuadrado $\left(\chi^{2}\right)$ es significativo, existe cierto consenso en la sensiblidad que tendría este test a factores como el tamaño muestral, siendo común obtener significación estadística en muestras grandes (>200) (Kline, 2011; Rose, Markman, \& Sawilowsky, 2017).

Respecto de las cargas factoriales, todos los ítems de las dimensiones que constituyen la EPE muestran altos pesos factoriales y significativos en los factores de primer orden (Ver Figura 1) y en el factor general, a excepción de dos ítems (ítem 6 e ítem 25).

Para evaluar el grado en que los datos pueden ser considerados con una estructura unidimensional global, se realizaron tres cálculos 
Tabla 4. Cargas factoriales para la escala de participación escolar

\begin{tabular}{|c|c|c|c|c|}
\hline Ítem & $\mathrm{DN}$ & $\mathrm{EE}$ & $\mathrm{AE}$ & PP \\
\hline 1 & $.533(.021)$ & & & \\
\hline 2 & $.823(.012)$ & & & \\
\hline 3 & $.857(.009)$ & & & \\
\hline 4 & $.863(.010)$ & & & \\
\hline 5 & $.800(.012)$ & & & \\
\hline 6 & $.563(.022)$ & & & \\
\hline 7 & & $.739(.015)$ & & \\
\hline 8 & & $.779(.013)$ & & \\
\hline 9 & & $.778(.013)$ & & \\
\hline 10 & & $.537(.021)$ & & \\
\hline 11 & & $.790(.013)$ & & \\
\hline 12 & & $.683(.017)$ & & \\
\hline 13 & & & $.809(.011)$ & \\
\hline 14 & & & $.864(.009)$ & \\
\hline 15 & & & $.820(.010)$ & \\
\hline 16 & & & $.773(.015)$ & \\
\hline 17 & & & $.717(.019)$ & \\
\hline 18 & & & $.655(.018)$ & \\
\hline 19 & & & $.793(.012)$ & \\
\hline 20 & & & & $.743(.017)$ \\
\hline 21 & & & & $.863(.009)$ \\
\hline 22 & & & & $.794(.012)$ \\
\hline 23 & & & & $.838(.010)$ \\
\hline 24 & & & & $.838(.010)$ \\
\hline 25 & & & & $.723(.15)$ \\
\hline Factor $\mathrm{G}$ & $.782(.015)$ & $.897(.013)$ & $.635(.020)$ & $.785(.015)$ \\
\hline
\end{tabular}

Nota. $\mathrm{N}=1428$; DN: Participación de las Decisiones y Normas escolares; EE: participación en los Eventos Escolares; AE: Participación en las Actividades Escolares (extra-académicas); PP: Percepción Positiva de la participación; Factor G: Participación Escolar General. ${ }^{a}$ Error estándar se incluye en el paréntesis.

Tabla 5. Estimaciones del modelo bifactorial de la escala de participación escolar

\begin{tabular}{cccccccc}
\hline & $\mathrm{X}^{2}$ & $\mathrm{~d} f$ & RMSEA & $(90 \% \mathrm{CI})$ & WRMR & CFI & TLI \\
\hline Modelo & $1412.286^{*}$ & 244 & .057 & $.054-$ & 1.374 & .970 & .963 \\
Bifactorial & & & .060 & & & \\
\hline Nota. $\mathrm{N}=1428 ;{ }^{*} p<.01$ & & & & & & &
\end{tabular}

adicionales mediante la calculadora de índices bifactoriales de Dueber (2017). Concretamente se utilizó el Porcentaje de correlaciones no contaminadas (PUC -percent of uncontaminated correlations) que representa el porcentaje de varianza que corresponde solo a la dimensión general, el porcentaje de varianza común explicada (ECV-explained common variance) que es la proporción de varianza total que es explicada por cada factor (general y específicos)- para los factores específicos el ECV refleja la fuerza de un factor específico para explicar la varianza de los ítems que cargan en él y el Omega jerárquico (OmegaH-omega Hierarchical), que refleja el porcentaje de varianza sistemática del puntaje total que puede ser atribuida a diferencias individuales en el factor general.

Los resultados de estos análisis adicionales indican que el PUC es relativamente alto (PUC=.78), muy cercano a lo recomendado
PUC >.80 (Reise, Scheines, Widaman \& Haviland, 2013). No obstante, el ECV es bajo para el factor general $(\mathrm{ECV}=.13)$, indicando sesgo relativo al forzar la estructura a una unidimensional y posee valores aceptables para los factores específicos $(\mathrm{DN}=.90 ; \mathrm{EE}=.89 ; \mathrm{AE}=.75 ; \mathrm{PP}=.93$ ) (Reise et al., 2013). Asimismo, el OmegaH indica un 3\% de variabilidad sistemática en el puntaje total puede ser atribuida únicamente a las diferencias individuales en el factor general y entre un $78 \%$ y $92 \%$ a los específicos.

Finalmente, para realizar la comparación de los modelos evaluados (factorial de segundo orden y bifactorial), se identificó la diferencia en los valores de chi-cuadrado y grados de libertad, para determinar del tamaño del efecto ( $\omega$ de Cohen). La solución del modelo bifactorial presentó un mejor ajuste de los datos que la solución presentada en el modelo de segundo orden. La magnitud de la diferencia del chi-cuadrado 


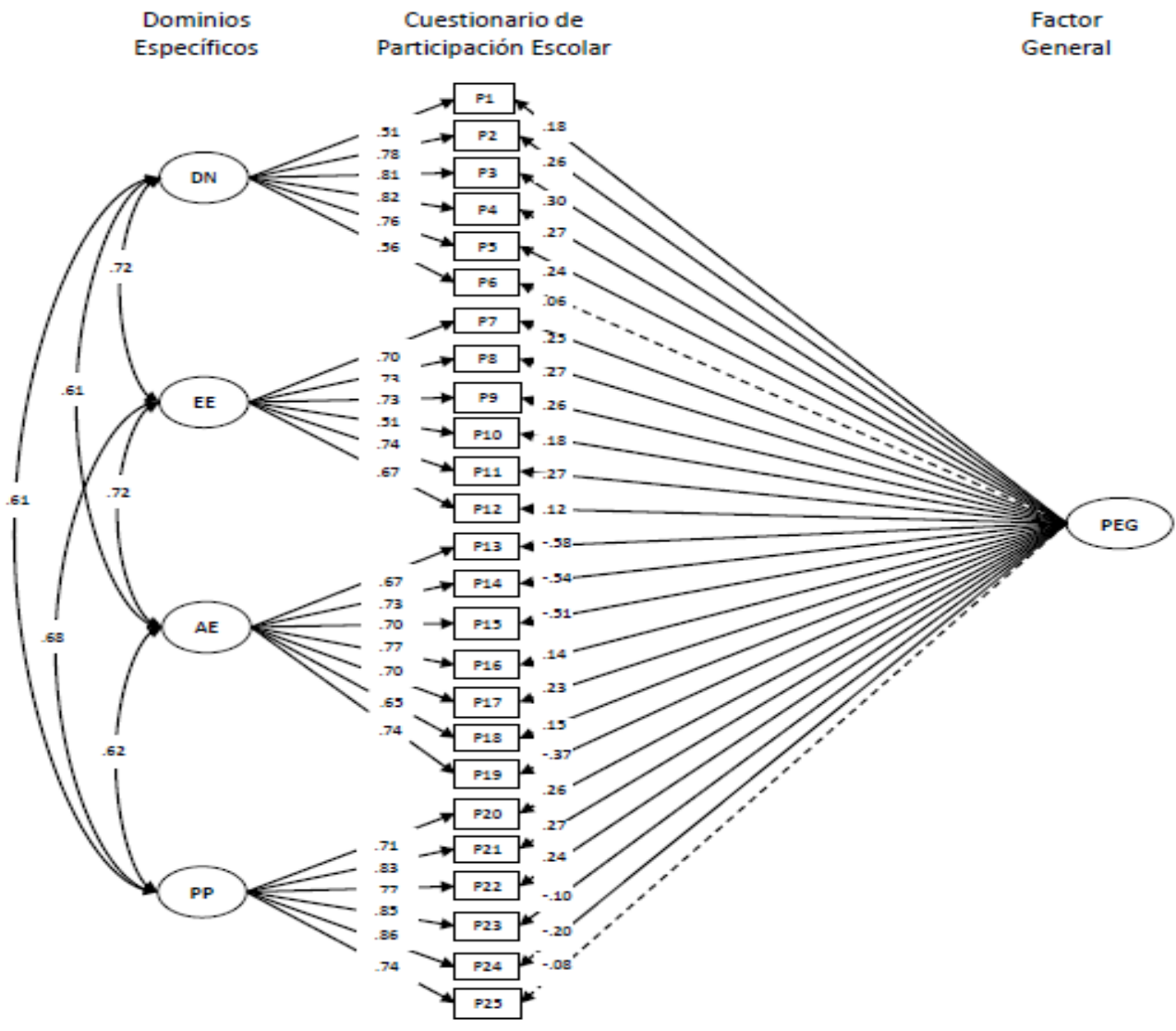

Figura 1. Diagrama de Estructura Bifactorial de la escala de participación escolar

Nota. DN: Participación de las Decisiones y Normas escolares; EE: participación en los Eventos Escolares; AE: Participación en las Actividades Escolares (extra-académicas); PP: Percepción Positiva de la participación; PEG: Participación Escolar General.

Tabla 6. Diferencias de Chi-cuadrado entre el modelo de segundo orden y el modelo bifactorial (comparación anidada) para la escala de Participación escolar

\begin{tabular}{|c|c|c|c|c|c|c|c|c|c|}
\hline & \multirow[t]{2}{*}{$X^{2}$} & \multirow[t]{2}{*}{$\mathrm{d} f$} & \multirow[t]{2}{*}{ RMSEA } & \multirow[t]{2}{*}{$(90 \% \mathrm{CI})$} & \multirow[t]{2}{*}{ WRMR } & \multirow[t]{2}{*}{ CFI } & \multicolumn{3}{|c|}{$\begin{array}{c}\text { Segundo orden Vs } \\
\text { Bifactorial }\end{array}$} \\
\hline & & & & & & & $\Delta \mathrm{x}^{2}$ & $\Delta \mathrm{d} f$ & $\omega$ \\
\hline $\begin{array}{l}\text { Modelo de } \\
\text { Segundo }\end{array}$ & $4422.529 *$ & 271 & .102 & .099-105 & 3.193 & .894 & & & \\
\hline Orden & & & & & & & 3010.243 & 27 & .28 \\
\hline $\begin{array}{l}\text { Modelo } \\
\text { Bifactorial }\end{array}$ & $1412.286^{*}$ & 244 & .057 & $.054-.060$ & 1.368 & .970 & & & \\
\hline
\end{tabular}

Nota. $\mathrm{N}=1428 ; * p<.01$

muestra la existencia de un tamaño del efecto mediano entre ambos modelos (ver Tabla 6).

\section{Discusión}

La escala de participación escolar presentó muy buenos índices de confiabilidad a través del coeficiente omega de McDonald, dando cuenta de una adecuada consistencia interna de sus dimensiones. Los resultados del análisis bifactorial confirman la estructura propuesta por los autores y es coherente con la naturaleza multidimensional del constructo así como con la interpretabilidad de un puntaje total (JohnAkinola \& Nic-Gabhainn, 2014). El modelo bifactorial demostró ser útil para captar esta configuración, al igual que en otras investigaciones que han testeado constructos similares (Steffansson et al., 2016).

No obstante lo anterior, es preciso aclarar que los índices de ajuste bifactoriales específicos calculados señalan que hay cierto sesgo al considerar la estructura como unidimensional. Esto porque la fuerza del factor general es menor 
que la de los factores específicos (ECV \& OmegaH), el porcentaje de correlación no contaminada (PUC) relativamente alto modera esta interpretación y hace que el valor ECV para el factor general sea menos relevantes para predecir el sesgo. Esto, a nuestro modo de ver, no invalida la interpretación del puntaje total de la escala de participación, pero sí lo haría si se descompusiera la varianza del puntaje total y solo se considerara la parte del puntaje total que correspondería al Factor General.

En términos generales, según Reise et al. (2013) al evaluar el ajuste de un modelo de medida unidimensional, el grado de sesgo de los coeficientes estructurales dependerá fuertemente y de manera inversa de la varianza común explicada, pero sus efectos estarán moderados por el porcentaje de correlaciones no contaminadas por la multidimensionalidad. De ahí, que estos autores señalen que cuando el PUC es alto (>.80) los coeficientes estructurales están relativamente no sesgados, incluso si la fuerza del factor general es baja en comparación a la fuerza de los factores específicos, es decir, "los valores de los índices de fuerza son menos importantes para predecir el sesgo" (p.22). Sin embargo, si bien se señalan valores de ECV y omegaH >.60 en estos casos, se reconoce que aún se requiere mayor investigación al respecto, para generar puntos de corte en diversas condiciones (número de ítems, número de factores).

En cuanto a las cargas factoriales, los únicos ítems que no tuvieron cargas significativas en el factor general fueron el ítem 6: "En mi colegio tenemos alumnos que representan a otros alumnos", perteneciente a la dimensión Participación en reglas y decisiones del colegio; y el ítem 25: "Estoy muy contento con mi nivel de participación en mi colegio", perteneciente a la dimensión Percepción positiva de la participación escolar. En el caso del primer ítem, una posible explicación puede ser que contar con un representante estudiantil no está significativamente asociado con el factor general, debido a que en ese caso la percepción global de participación de los alumnos es más bien indirecta, a través del representante y no ser percibida por los estudiantes como una participación global efectiva. En este sentido (Shnapper, 2000) señala que la idea de representación puede contraponerse con la idea de apertura de los derechos políticos para todos los miembros de un grupo, puesto que el "otro" jamás podrá "representarlos". Según Inzunza (2009), esto es lo que ha ido sucediendo en los estudiantes secundarios Chilenos, quienes paulatinamente han dejado de creer en instituciones como los centros de estudiantes tradicionales y sus representantes, y han dado paso otros tipos de asociatividad más informales y de tipo asambleario.

En la misma línea de argumentación, el ítem 25 puede que no cargue significativamente en el factor general de participación porque está midiendo qué tan "contento" está el estudiante con su nivel de participación en la escuela. Esto puede no necesariamente estar relacionado con su percepción global de las oportunidades de participación que brinda la escuela en todos los otros aspectos evaluados.

Con respecto a los ítems que presentaron cargas negativas en el factor general, se puede deber a diferencias transculturales que se presentan cuando se adapta un instrumento de un país a otro. Las cargas negativas de los ítems 13, 14, 15 y 19 que corresponden a la dimensión "Participación en actividades escolares (extraacadémicas)" pueden estar reflejando dificultades en los escolares chilenos para evaluar los distintos tipos de actividades extra-académicas propuestos para esta dimensión por John-Akinola y NicGabhainn (2014). Así, en Chile, la educación física y las clases de arte se realizan como parte del currículo escolar y, por tanto, siguen una determinada estructura. Por otra parte, no todos los colegios ofrecen cursos de teatro o música y, en algunos casos, estos se realizan fuera del colegio. Finalmente, las cargas negativas de los ítems 23 y 24 que corresponden a "Percepción positiva de la participación" pueden responder a que estos evalúan el grado en que la participación en sus colegios es "divertida" o los "hace sentirse positivos". Al parecer, los escolares no asocian estas reacciones emocionales, ya sea porque las actividades en las que participan no les parecen atractivas o porque, tal y como pueden estar concebidas en sus colegios, generan otras emociones (v.g., respeto).

Los valores de participación en este estudio fueron superiores a los obtenidos en la muestra de validación inicial del cuestionario de participación 
(John-Akinola \& Nic-Gabhainn, 2014). Los participantes de esta investigación mostraron niveles relativamente altos de participación en las decisiones y reglas en su escuela en los eventos y en las actividades escolares (extra-académicas), con excepción de la percepción positiva de la participación. Lo anterior es coherente con lo recién argumentado respecto al ítem 25 y podría deberse, quizás a una actitud crítica de los estudiantes respecto al nivel de participación ejercido y a las implicancias que puede tener esta participación en la escuela. También se puede relacionar a los importantes procesos de reforma educacional en Chile y activismo por parte de los estudiantes secundarios que se han vivido en la última década en este país (Inzunza, 2009).

Los altos niveles de participación encontrados en este estudio, contrastan con la baja participación política de jóvenes reportada por encuestas representativas realizadas en Chile, donde el $81 \%$ de los jóvenes declara no estar interesado en la política y el $58.1 \%$ de ellos reporta no haber votado en las últimas elecciones presidenciales (Instituto Nacional de la Juventud [INJUV], 2017). Sin embargo, el 50\% de la población joven ha participado en al menos una organización y el $28 \%$ en alguna actividad de ayuda a la comunidad. Finalmente, el 23\% declara haber participado en alguna acción de carácter no convencional durante el último año, caracterizadas por el asociacionismo y solución de problemas comunitarios (INJUV, 2017). En este sentido, la participación en la escuela, especialmente si consideramos la carga no significativa del ítem relacionado al representante estudiantil, quizás alude a que la escuela es ser percibida por los jóvenes como una posibilidad de participación no convencional, pero válida.

La utilidad de evaluar el constructo participación escolar a través del modelado bifactorial radica en una comprobación más precisa de la estructura factorial propuesta $y$, sobre todo, por la posibilidad de contar con un puntaje total interpretable. Esto es de relevancia para la investigación, puesto que avala la utilización del constructo participación como un todo y su relación con otras variables dentro de un modelo teórico a testear. En consecuencia, la EPE puede resultar útil para la investigación de este constructo en la escuela y analizar este fenómeno de manera confiable y válida en el contexto chileno.

En cuanto a las limitaciones de este estudio, se encuentran la evaluación de constructo de la escala de participación escolar, solo a través de análisis factorial confirmatorio y no con otras escalas que permitieran evaluar la validez convergente y divergente. Esto se puede explicar desde la ausencia de instrumentos validados en el país evaluando este concepto. No obstante lo anterior, sería interesante para futuras investigaciones relacionar los resultado obtenidos con el instrumento aquí presentado y su relación predictiva con otras variables testeadas en otras investigaciones tales como el clima escolar positivo, bienestar subjetivo y conductas prosociales (de Róiste, Kelly, Molcho, Gavin, \& Gabhainn, 2012; Finn \& Rock, 1997; JohnAkinola \& Nic-Gabhainn, 2014), la relación de la participación escolar con las actitudes hacia la autoridad (Gálvez-Nieto, Vera-Bachman, Trizano, \& García, 2015) o su relación con la participación de los apoderados (Pérez-Villalobos, Jiménez, Vilos, \& Ortiz, 2015).

\section{Agradecimientos}

Este artículo contó con financiamiento CONICYT. Proyecto Fondecyt Regular 1151360: Reconocimiento, respeto y participación de minorías: Una investigación en escolares chilenos, Mapuche, inmigrantes y con discapacidad. 20152017.

\section{Referencias}

Brouwer, D., Meijer, R. R., Weekers, A. M., \& Baneke, J. J. (2008). On the dimensionality of the dispositional hope scale. Psychological Assessment, 20(3), 310.

Brown, T. A. (2014). Confirmatory factor analysis for applied research ( $2 \mathrm{~d}$ ed.). New York: Guilford Publications.

Chen, F. F., Jing, Y., Hayes, A., \& Lee, J. M. (2013). Two concepts or two approaches? A bifactor analysis of psychological and subjective well-being. Journal of Happiness Studies, 14(3), 1033-1068.

Chen, F. F., West, S. G., \& Sousa, K. H. (2006). A comparison of bifactor and second-order 
models of quality of life. Multivariate Behavioral Research,

Cornejo, R., \& Redondo, J. M. (2001). El clima escolar percibido por los alumnos de enseñanza media: Una investigación en algunos liceos de la Región Metropolitana. Última década, 9(15), 11-52.

Cucina, J., \& Byle, K. (2017). The bifactor model fits better than the higher-order model in more than $90 \%$ of comparisons for mental abilities test batteries. Journal of Intelligence, 5(3), 27, $1-21$.

de Róiste, A., Kelly, C., Molcho, M., Gavin, A., \& Nic Gabhainn, S. (2012). Is school participation good for children? Associations with health and wellbeing. Health Education, 112(2), 88-104.

Dueber, D. M. (2017). Bifactor indices calculator: A Microsoft excel-based tool to calculate various indices relevant to bifactor CFA models. https://dx.doi.org/10.13023/edp.tool.01

Finn, J. D., \& Rock, D. A. (1997). Academic success among students at risk for school failure. Journal of Applied Psychology, 82(2), 221.

Fullarton, S. (2002). Student engagement with school: Individual and school-level influences. Recuperado el 16 de abril del 2017 de

http://www.acer.edu.au/documents/LSAY_ex ecsum27.pdf

Gade, J. C., Schermelleh-Engel, K., \& Klein, A. G. (2017). Disentangling the common variance of perfectionistic strivings and perfectionistic concerns: A bifactor model of perfectionism. Frontiers in Psychology, 8, 13. doi:10.3389/fpsyg.2017.00160

Gálvez-Nieto, J. L., Vera-Bachman, D., Trizano, Í., \& García, J. A. (2015). Examen psicométrico de la Escala de Actitudes hacia la Autoridad Institucional (AAI-A), en estudiantes chilenos. Revista Iberoamericana de Diagnóstico y Evaluación - e Avaliação Psicológica, 1(39), 57-67.

Geiser, C. (2012). Data analysis with Mplus: Guilford Press.

Gignac, G. E. (2016). The higher-order model imposes a proportionality constraint: That is why the bifactor model tends to fit better.
Intelligence,

55, $57-68$. doi:http://dx.doi.org/10.1016/j.intell.2016.01.006

Griebler, U., Rojatz, D., Simovska, V. \& Forster, R. (2017). Effects of student participation in school health promotion: A systematic review. Health Promotion International. doi:10.1093/heapro/dat090.

Hindman, A. H., Pendergast, L. L., \& Gooze, R. A. (2016). Using bifactor models to measure teacher-child interaction quality in early childhood: Evidence from the Caregiver Interaction Scale. Early Childhood Research Quarterly, 36, 366-378. doi:10.1016/j.ecresq.2016.01.012

Holzinger, K. J., \& Swineford, F. (1937). The bifactor method. Psychometrika, 2(1), 41-54.

Instituto Nacional de la Juventud. (2017). Octava encuesta nacional de la juventud 2015. Santiago, Chile: Ministerio de Desarrollo Social.

Inzunza, J. (2009). La asociatividad juvenil en los liceos públicos chilenos: la disolución de los centros de alumnos. En J. Redondo \& L. Muñoz (Eds.), Juventud y enseñanza media en Chile del Bicentenario. Antecedentes de la revolución pingüina (pp. 377-393). Santiago, Chile: OPECH.

JASP Team (2018). JASP (Version 0.8.4) [Computer software] (BibTex).

John-Akinola, Y. O., Gavin, A., O’Higgins, S. E., \& Nic Gabhainn, S. (2014). Taking part in school life: Views of children. Health Education, 114(1), 20-42

John-Akinola, Y. O., \& Nic-Gabhainn, S. (2014). Children's participation in school: A crosssectional study of the relationship between school environments, participation and health and well-being outcomes. BMC Public Health, 14(1), 964-974.

Kline, R. B. (2011). Principles and practice of structural equation modeling (3rd ed.). New York: The Guilford Press.

Libbey, H. P. (2004). Measuring student relationships to school: Attachment, bonding, connectedness, and engagement. Journal of School Health, 74(7), 274-283.

Luján-Tangarife, J., \& Cardona-Arias, J. (2015). Construcción y validación de escalas de medición en salud: Revisión de propiedades 
psicométricas. Archivos de Medicina, 11(3), 1-10.

Michaelides, M. P., Zenger, M., Koutsogiorgi, C., Brahler, E., Stobel-Richter, Y., \& Berth, H. (2016). Personality correlates and gender invariance of wording effects in the German version of the Rosenberg Self-Esteem Scale. Personality and Individual Differences, 97, 13-18. doi:10.1016/j.paid.2016.03.011

Ministerio de Educación de Chile. (2014). Informe nacional: Educación para todos en Chile. Santiago: Autor.

Ministerio de Educación de Chile. (2017). Estadísticas de la Educación 2016. Santiago: Autor.

Muñiz, J., Elosua, P., \& Hambleton, R. K. (2013). Directrices para la traducción y adaptación de los tests: Segunda edición. Psicothema, 25(2), 151-157.

Muthén, L., \& Muthén, B. (2013). Mplus 7.11. Los Angeles, CA: Autor.

Pérez-Villalobos, C., Jiménez, J., Vilos, V., \& Ortíz, H. (2015). Análisis de las Propiedades Psicométricas de la Escala de Satisfacción con la Gestión Escolar para Padres. Revista Iberoamericana de Diagnóstico y Evaluación - e Avaliação Psicológica, 1(39), 34-45.

Reise, S.P., Moore, T., \& Haviland, M, (2010). Bifactor models and rotations: Exploring the extent to which multidimensional data yield univocal scale scores, Journal of Personality Assessment, 92(6), 544-550.

Reise, S. P., Scheines, R., Widaman, K. F., \& Haviland, M. G. (2013). Multidimensionality and structural coefficient bias in structural equation modeling a bifactor perspective. Educational and Psychological Measurement, 73(1), 5-26.

Rodríguez, M., Kohen, R., Delval, J., \& Messina, C. (2016). De la escuela democrática a la participación política y ciudadana. Cultura y Educación, 28(1), 99-129.

Rose, S. A., Markman, B., \& Sawilowsky, S. (2017). Limitations in the systematic analysis of structural equation model fit indices. Journal of Modern Applied Statistical Methods, 16(1), 69-85.

Scherer, R., Nilsen, T., \& Jansen, M. (2016). Evaluating individual students' perceptions of instructional quality: An investigation of their factor structure, measurement invariance, and relations to educational outcomes. Frontiers in Psychology, 7, 16. doi:10.3359/fpsyg.2010.00110

Schult, J., \& Sparfeldt, J. R. (2016). Do non-g factors of cognitive ability tests align with specific academic achievements? A combined bifactor modeling approach. Intelligence, 59, 96-102. doi:10.1016/j.intell.2016.08.004

Schnapper, D. (2000). Qu'est-ce que la citoyenneté?. Gallimard: Francia.

Simovska, V. (2004). Student participation: A democratic education perspective. Experience from the health-promoting schools in Macedonia. Health Education Research, 19(2), 198-207.

Simovska, V. (2012). What do health-promoting schools promote? Processes and outcomes in school health promotion. Health Education, 122(2), 84-87.

Stefansson, K. K., Gestsdottir, S., Geldhof, G. J., Skulason, S., \& Lerner, R. M. (2016). A bifactor model of school engagement: Assessing general and specific aspects of behavioral, emotional and cognitive engagement among adolescents. International Journal of Behavioral Development, 40(5), 471-480. doi:10.1177/0165025415604056

Ventura-León, J. L., \& Caycho-Rodríguez, T. (2017). El coeficiente omega: Un método alternativo para la estimación de la confiabilidad. Revista Latinoamericana de Ciencias Sociales, Niñez y Juventud, 15(1), 625-627. 